\title{
INTERNACIONALIZAÇÃO, INOVAÇÃO, EMPREENDEDORISMO E SUA RELAÇÃO COM DESEMPENHO ECONÔMICO DE CIDADES: UM ESTUDO COM CIDADES DO ESTADO DE SÃO PAULO
}

\section{Beatriz Garcia ${ }^{1}$}

Cristiano Morini ${ }^{1}$

Daniel Henrique Dario Capitani ${ }^{1}$

\footnotetext{
${ }^{1}$ Universidade Estadual de Campinas
} 


\title{
INTERNACIONALIZAÇÃO, INOVAÇÃO, EMPREENDEDORISMO E SUA RELAÇÃO COM DESEMPENHO ECONÔMICO DE CIDADES: UM ESTUDO COM CIDADES DO ESTADO DE SÃO PAULO
}

\begin{abstract}
Resumo: Embora existam muitas pesquisas com temas relacionados à internacionalização, empreendedorismo e inovação no contexto das firmas, pouco tem sido estudado no sentido de entender o comportamento dessas variáveis no contexto das cidades. O objetivo desta pesquisa é analisar qual a relação entre as dimensões internacionalização, inovação e empreendedorismo no contexto das cidades, no sentido de geração de riqueza e aumento do nível de emprego. Não foram identificados estudos existentes que tratem destas dimensões em conjunto. Pesquisas podem ser úteis para subsidiar a formulação de políticas públicas e iluminar novas formas de análises da governança das cidades. Por meio de Mínimos Quadrados Ordinários (MQO) com base de dados secundários de 35 municípios do Estado de São Paulo, foram analisadas qual a relação das dimensões de pesquisa. Por meio das regressões lineares múltiplas, verificou-se que há influência das dimensões internacionalização, inovação e empreendedorismo sobre o desempenho econômico, sobretudo nas taxas de emprego.
\end{abstract}

Palavras-chave: município. Internacionalização. Empreendedorismo. Inovação.

\section{$1 \quad$ Introdução}

Com as transformações econômicas mundiais e aumento da concorrência, as organizações desenvolvem estratégias capazes de garantir vantagens competitivas e a sua sobrevivência no mercado (BOERMANS; ROELFSEMA, 2016). Estudos recentes têm utilizado a internacionalização também como estratégia para endereçar problemas urbanos. Pierre (2019) afirma que instituições na Europa cada vez mais têm influenciado umas às outras, enquanto o governo tem direcionado sua atenção para as leis e monitoramento de sua aplicação.

O modelo conceitual criado por Boermans e Roefsema (2016) mostra a interação mútua entre internacionalização e inovação e seus efeitos positivos sobre o crescimento das vendas e empregos. De acordo com Veglio e Zucchella (2015), a relação entre internacionalização e inovação é justamente a essência dos estudos sobre empreendedorismo internacional (EI) (VEGLIO; ZUCCHELLA, 2015). Conforme Castaño et al. (2016), uma das características mais importantes para o empreendedor se estabelecer no mercado nacional e internacional é a inovação. Oparoacha (2015), por sua vez, afirma que, independente do momento em que a firma internacionaliza suas atividades, o EI sempre envolve características inovadoras, exploração de oportunidades e estratégias competitivas em mercado internacional. Segundo Castaño et al. (2016), o empreendedorismo também é fonte de riqueza e emprego, devido à capacidade de empreendedores combinarem recursos e introduzirem produtos inovadores no mercado. Desta forma podemos notar as três dimensões (internacionalização, inovação e empreendedorismo) interligadas.

Conforme constatado por Oura et al. (2016), estudos realizados até o momento para países em desenvolvimento, considerando as dimensões internacionalização, empreendedorismo e inovação, estão predominantemente voltados para a China (AHLSTROM 
et al., 2018; RIALP-CRIADO; KOMOCHKOVA, 2017; WU et al., 2016; ZHANG et al., 2016). A presente pesquisa considera estas três dimensões no contexto brasileiro.

Com base nas informações apresentadas - existência de poucos estudos que explorem atividades internacionais, inovadoras e empreendedoras em municípios brasileiros - e considerando a importância de formuladores de políticas públicas possuírem insumos para compreenderem a importância de atividades internacionais, empreendedoras e inovadoras para o desempenho econômico das cidades, foi elabora a seguinte pergunta de pesquisa: Há relação entre estas dimensões no contexto das cidades? Há estímulos por parte das cidades para que estas dimensões sejam alavancadas? Como?

\section{$2 \quad$ Referencial Teórico}

A seleção do portfólio bibliográfico foi feita em três etapas e utilizou o método da revisão sistemática da literatura, via ProKnow-C (ENSSLIN, et al., 2010), a fim de selecionar literatura relevante e encontrar as lacunas de pesquisa. A escolha do método justifica-se pela praticidade em fazer o levantamento e a organização de portfólio bibliográfico, por possibilitar a construção de conhecimento e fornecer detalhes da pesquisa que possam auxiliar pesquisadores da área em investigações futuras.

A fim de contribuir com pesquisadores das áreas de internacionalização, inovação e empreendedorismo, os 49 artigos selecionados na primeira etapa foram listados e numerados no referencial bibliográfico deste trabalho. A Figura 1 apresenta como os artigos estão distribuídos entre as três dimensões (internacionalização, inovação e empreendedorismo).

Com a leitura dos artigos notou-se que $100 \%$ tratavam de ao menos um dos aspectos seguintes: visão baseada em recursos (VBR); abordagem de redes; e, importância das instituições. A Figura 2 apresenta quais artigos trazem um ou mais desses aspectos, seja na introdução, na revisão bibliográfica, na metodologia, ou nos resultados.

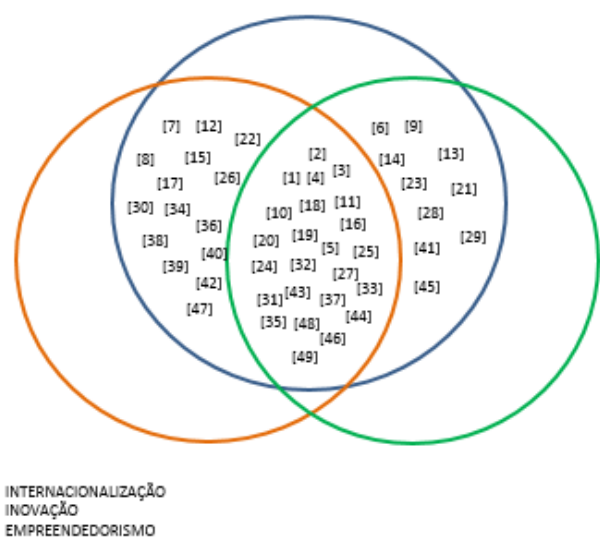

Figura 1 - Distribuição do banco de artigos entre as dimensões internacionalização, inovação e empreendedorismo

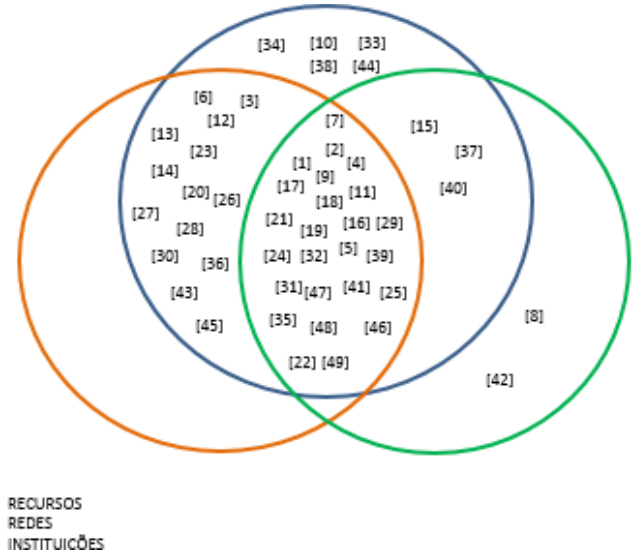

Figura 2 - Distribuição do banco de artigos entre os aspectos recursos, redes e instituições

\subsection{Breve contextualização da internacionalização}

Em 1977, Johanson e Vahlne (1977) propõem um modelo baseado em observações empíricas realizadas em estudos na Universidade de Uppsala. Os dados mostram que as 
organizações desenvolvem as atividades internacionais por etapas ao invés de realizarem grandes investimentos iniciais. Além disso, mencionam a progressão geográfica dos negócios internacionais sob o efeito da distância psicológica do mercado alvo, e que o processo de internacionalização ocorre com base em experiências e aprendizados obtidos de mercados externos (JOHANSON; VAHNE, 1977).

A partir dos anos 1980, nota-se que o processo de internacionalização passou a apresentar mudanças de caráter evolutivo e conceitual. O que no início representava estratégia realizada de forma gradativa ou esporádica passa a ser uma justificativa estratégica desde o nascimento da organização. É nesse momento que se dá origem à terminologia "nascidas globais" (born globals) - empresas que nascem com o objetivo de atender o mercado internacional (OVIATT; McDOUGALL, 1994).

A partir de então, busca-se entender outras variáveis e suas influências na internacionalização das firmas: velocidade da internacionalização (qual o timming de internacionalização das empresas), denominado por alguns autores como "idade" da empresa no momento da internacionalização (BASTIAN; TUCCI, 2017; BAUM; SCHWENS; KABST, 2015; KURYAN; KHAN; GUSTAFSSON, 2018; MUSTEEN; DATTA; BUTTS, 2014); tamanho da empresa, sendo pequenas e médias empresas ou empresas tradicionais (AHLSTROM et al., 2018; ALVES et al., 2017; BAUM; SCHWENS; KABST, 2015; BRUNEEL; CLARYSSE; AUTIO, 2018; FELZENSZTEIN et al., 2015; HERSTAD; EBERSBERGER, 2015; KURYAN; KHAN; GUSTAFSSON, 2018; VANNINEN; KUIVALAINEN; CIRAVEGNA, 2017); e, formas de entrada (exemplo, subsidiárias, joinventures, etc.) (HAGEN; ZUCCHELLA, 2014; WU et al., 2016).

\subsection{Internacionalização e sua relação com a Inovação}

Desde a criação do conceito de inovação como um processo de "criação destrutiva" (SCHUMPETER, 1952), houve diversas modificações e classificações distintas para o conceito (CHIVA; GHAURI; ALEGRE， 2014; CUERVO-CAZURRA; RAMAMURTI，2017; LEWANDOWSKA; SZYMURA-TYC; GOŁEBIOWSKI, 2016; RASK, 2014). O mais utilizado pela literatura (CHIVA; GHAURI; ALEGRE, 2014; HERSTAD; EBERSBERGER, 2015; LEWANDOWSKA; SZYMURA-TYC; GOLEBIOWSKI, 2016; OURA; ZILBER; LOPES, 2016; PRANGE; PINHO, 2017; RODIL; VENCE; SÁNCHEZ, 2016; SIEDSCHLAG; ZHANG, 2015) tem sido o do Manual de Oslo (OCDE, 2005), o qual classifica a inovação como a implementação de um produto ou processo novo ou significativamente melhorado. Sendo que a inovação desenvolvida, ou adotada, pode ser nova para o mundo ou para a empresa.

Chiva et al. (2014) afirmam que a internacionalização, além de ser fonte valiosa de aprendizado, é também fonte de novas ideias que surgem de outros mercados com diferentes culturas e necessidades, e que consequentemente podem estimular a inovação. Para Lewandowska et al. (2016), a internacionalização e a inovação são fenômenos interrelacionados para economias e firmas. O levantamento bibliográfico feito por Veglio \& Zucchela (2015) constataram que, para alguns autores, a internacionalização em si é vista como uma expressão de inovação.

\subsection{Internacionalização e sua relação com o Empreendedorismo}


O empreendedorismo, sob a ótica de Schumpeter (1988), está associado à identificação e ao aproveitamento de novas oportunidades no contexto dos negócios. Conforme Baron (2010) trata-se de um conceito formado pelo conjunto de pessoas e processo, no qual o empreendedor segue as etapas para tornar ideias em oportunidades, e posteriormente em negócios de sucesso.

O empreendedorismo tem sido visto pela literatura como importante fator de crescimento e prosperidade para economia de um país (CASTAÑO; MÉNDEZ; GALINDO, 2016).

A internacionalização tem sido um atrativo para empreendedores, uma vez que traz a possibilidade de novos mercados, crescimento (CASTAÑO; MÉNDEZ; GALINDO, 2016), e impactos positivos em inovação (BOERMANS; ROELFSEMA, 2016). Para Oparaocha (2015), a exploração de oportunidades internacionais é vital para o crescimento do empreendedorismo. Ao ser analisada a relação contrária, pode-se dizer que a orientação empreendedora é um atributo que ajuda a firma a superar os obstáculos do processo de internacionalização (DAI et al., 2014; ZHANG et al., 2016).

\subsection{A Teoria Baseada em Recursos, Abordagem de redes, Teoria institucional e sua} importância para a internacionalização, empreendedorismo e inovação

Lewandowska et al. (2016) afirmam que a abordagem de redes tem dominado os modelos recentes de inovação e internacionalização de firmas. No caso de países emergentes, como o Brasil, existe uma tendência de empresas (principalmente as pequenas e médias) possuírem recursos limitados (OURA; ZILBER; LOPES, 2016), o que pode dificultar os processos de internacionalização e inovação. Boermans \& Roefsema (2016) afirmam que os recursos possuem efeito moderador sobre internacionalização, uma vez que apresentou alta correlação com internacionalização e crescimento de empregos e vendas.

Considerando que as firmas atuam em ambientes institucionais que definem e controlam a maneira como elas operam (por meio de leis, cultura, mercados financeiros, regulamentações, políticas, padrões industriais, educação, etc.) é importante que se estabeleçam relações institucionais, a fim de buscar suporte para as suas operações, investimentos internacionais, minimizar riscos (OPARAOCHA, 2015), diminuir barreiras para exportação e gerar apoios financeiros e não financeiros (DÍAZ-ARIZA; GARCÍA-CASTIBLANCO; MUÑOZ, 2019). Do contexto das firmas para o contexto das cidades, a literatura destaca a atenção que tem sido dada para as instituições que fazem interligações entre cidades do mesmo país e de países distintos (PIERRE, 2019).

Com as comprovações empíricas da importância do apoio institucional para o EI (OPARAOCHA, 2015), deve ser de interesse do governo promover e incentivar apoios institucionais (governamentais ou não) para as firmas e cidades a fim de garantir sua competitividade internacional. O que converge com as afirmações de Baum et al. (2015).

Lewandowska et al. (2016) ressaltam a importância de relacionamentos com universidades e clientes, afirmando que essas relações podem promover maiores chances de inovação de produto, do que a parceria com somente um tipo de instituição.

$2.5 O$ círculo virtuoso entre internacionalização, inovação, empreendedorismo e o desempenho das cidades 
A revisão teórica feita para elaboração deste estudo identificou a possível existência de relação mútua e positiva entre as dimensões: internacionalização, inovação e empreendedorismo.

Pierre (2019) afirma que cidades da Europa, por meio de instituições, participam de projetos internacionais e tendem a estabelecer rede de contatos que compartilham problemas e soluções por meio do aprendizado em conjunto. Mais do que isso, o autor levantou que governos locais da Suécia têm utilizado a internacionalização para obter capacidade de endereçar problemas de mudança climática e sustentabilidade e outros problemas.

Sá Porto \& Rocha (2017) defendem a importância dos formuladores de políticas locais em entender e utilizar fatores considerados importantes pelas firmas, a fim de buscar políticas para melhorar o ambiente de negócios local e promover o desenvolvimento econômico local.

Hipótese: Internacionalização, empreendedorismo e inovação têm efeitos positivos sobre o desempenho das cidades.

As fontes de informações e rankings de cidades encontrados durante a pesquisa foram relacionadas e listadas no Quadro 1.

FONTES DE INFORMAÇÕES OU RANKINGS DE CIDADES E/OU REGIÕES

\begin{tabular}{|c|c|c|c|}
\hline Nome & Dimensões & Endereço & $\begin{array}{l}\text { Cidades/regi } \\
\text { oes } \\
\text { brasileiras }\end{array}$ \\
\hline $\begin{array}{l}\text { China Stock Market \& } \\
\text { Accounting Research } \\
\text { (CSMAR) }\end{array}$ & Informações Financeiras & http://www.ceibs.edu/ & Não \\
\hline $\begin{array}{l}\text { Community Innovation } \\
\text { Survey (CIS) }\end{array}$ & $\begin{array}{l}\text { Economia e finanças; } \\
\text { População e Condições sociais; } \\
\text { Indústria, comércio e serviços; } \\
\text { Agricultura; Comércio } \\
\text { internacional; Transporte; Meio } \\
\text { ambiente e energia; Ciência, } \\
\text { Tecnologia e sociedade digital }\end{array}$ & $\begin{array}{l}\text { https://ec.europa.eu/eurostat/web/mic } \\
\text { rodata/community-innovation-survey }\end{array}$ & Não \\
\hline $\begin{array}{l}\text { CSC - Connected } \\
\text { SmartCities }\end{array}$ & $\begin{array}{l}\text { Empreendedorismo; Inovação; } \\
\text { Mobilidade; Segurança; } \\
\text { Economia; Energia; } \\
\text { Governança; Saúde; Educação; } \\
\text { Meio ambiente; Tecnologia; } \\
\text { Urbanismo }\end{array}$ & $\begin{array}{l}\text { http://www.connectedsmartcities.co } \\
\text { m.br }\end{array}$ & Sim \\
\hline Dataviva & $\begin{array}{l}\text { Internacionalização (importação } \\
\text { e exportação); Atividades } \\
\text { econômicas; Universidades; } \\
\text { Balança Comercial (por tipo de } \\
\text { produto); Cursos básicos }\end{array}$ & http://dataviva.info/pt/ & Sim \\
\hline $\begin{array}{l}\text { Global Entrepreneurship } \\
\text { Monitor (GEM) }\end{array}$ & $\begin{array}{l}\text { Comportamentos e atitudes } \\
\text { empreendedoras; Condições } \\
\text { nacionais para o } \\
\text { empreendedorismo }\end{array}$ & https://www.gemconsortium.org/data & Sim \\
\hline $\begin{array}{l}\text { Índice de Potencial } \\
\text { Inovador }\end{array}$ & $\begin{array}{l}\text { Meio ambiente/urbanismo; } \\
\text { Sócio_demográfico; Fiscal e } \\
\text { Institucional; Econômico; } \\
\text { Inovação }\end{array}$ & $\begin{array}{l}\text { City size and competitiveness for the } \\
\text { cities of São Paulo: a spatial statistics } \\
\text { analysis - Sá Porto e Rocha (2017) }\end{array}$ & Sim \\
\hline Índice Firjan & $\begin{array}{l}\text { Emprego; Renda; Educação;e } \\
\text { Saúde }\end{array}$ & https://www.firjan.com.br/ifdm/ & Sim \\
\hline
\end{tabular}




\begin{tabular}{|c|c|c|c|}
\hline $\begin{array}{l}\text { Instituto Nacional de } \\
\text { Propriedade e Industrial } \\
\text { (INPI) }\end{array}$ & $\begin{array}{l}\text { Marca; Patente; Desenho } \\
\text { Industrial; Indicação } \\
\text { Geográfica; Programa de } \\
\text { computador; Topografia de } \\
\text { circuito; Transferência de } \\
\text { tecnologia; Informação } \\
\text { Tecnológica }\end{array}$ & http://www.inpi.gov.br/ & Sim \\
\hline $\begin{array}{l}\text { Investe São Paulo - } \\
\text { Agencia Paulista de } \\
\text { Promoção de Investimento } \\
\text { e Competitividade }\end{array}$ & $\begin{array}{l}\text { Divisões territoriais; } \\
\text { Infraestrutura; Educação; } \\
\text { Pesquisa e Inovação }\end{array}$ & http://www.investe.sp.gov.br & Sim \\
\hline Meu município & $\begin{array}{l}\text { Receitas e Despesas; e } \\
\text { Indicadores Gerenciais }\end{array}$ & https://meumunicipio.org.br & Sim \\
\hline Portal do empreendedor & $\begin{array}{l}\text { Empreendedorismo (quantidade } \\
\text { de MEIs por CNAE, município, } \\
\text { etc) }\end{array}$ & $\begin{array}{l}\text { http://www.portaldoempreendedor.g } \\
\text { ov.br/ }\end{array}$ & Sim \\
\hline $\begin{array}{l}\text { SEADE - IMP } \\
\text { (Informação dos } \\
\text { Municípios Paulistas) }\end{array}$ & $\begin{array}{l}\text { População e estatísticas vitais; } \\
\text { Educação; Saúde; Trabalho; } \\
\text { Condições de vida; Economia }\end{array}$ & http://www.imp.seade.gov.br & Sim \\
\hline $\begin{array}{l}\text { Serviço Brasileiro de } \\
\text { Apoio às Micro e } \\
\text { Pequenas Empresas } \\
\text { (SEBRAE) }\end{array}$ & $\begin{array}{l}\text { Empreendedorismo; } \\
\text { Planejamento; Finanças; } \\
\text { Mercado e Vendas; Inovação; } \\
\text { Cooperação; Pessoas; } \\
\text { Organização; Leis }\end{array}$ & $\begin{array}{l}\text { http://www.sebrae.com.br/sites/Porta } \\
\text { lSebrae }\end{array}$ & Sim \\
\hline
\end{tabular}

Quadro 1 - Fontes de informações ou rankings de cidades e/ou regiões

\section{METODOLOGIA}

\subsection{Seleção da amostra}

O contexto brasileiro foi selecionado para realização dessa pesquisa pelas principais razões: conforme constatado por Oura et al. (2016), pesquisas sobre atividades internacionais em países emergentes geralmente estão sendo realizadas na China (AHLSTROM et al., 2018; RIALP-CRIADO; KOMOCHKOVA, 2017; WU et al., 2016; ZHANG et al., 2016). No banco de artigos levantados na etapa 1 de pesquisa, dentre os 49 artigos selecionados utilizando a metodologia ProKnow-C foi encontrado apenas um artigo realizado no contexto brasileiro e nas etapas 2 e 3, poucos trabalhos foram encontrados no contexto brasileiro (NUNES; STEINBRUCH, 2019; GUIMARÃES, S. K.; AZAMBUJA, L. R., 2018; JESUS, 2017).

Quanto à escolha do estado de São Paulo, a razão se dá pelo fato de ser o estado brasileiro que fornece grande parte de bens de consumo, bens de capital, insumos e serviços para as demais regiões do Brasil, a além de ser o principal estado exportador do país (INVESTE SÃO PAULO, 2018).

Para selecionar as cidades do Estado de São Paulo foi considerada base de informações concedidas pela Investe SP (2018) - Agência Paulista de Promoção de Investimentos e Competitividade. No site da Investe SP é disponibilizada a ferramenta "Mapa SP Conecta", a qual é administrada pela Investe São Paulo e que apresenta as organizações que querem contribuir e integrar o ecossistema de apoio à inovação e ao empreendedorismo do estado. Para fazer parte desse ecossistema, a organização deve se cadastrar no site da Investe SP e informar em qual categoria se enquadra. Considerando as dimensões desta pesquisa, foram selecionadas 
as cidades com a presença de ao menos um desses requisitos: parques tecnológicos, incubadoras, e centros de $\mathrm{P} \& \mathrm{D}$ e/ou institutos de pesquisa.

Como resultado foram obtidas as 19 cidades: Americana, Araraquara, Atibaia, Botucatu, Campinas, Lins, Marília, Mogi das Cruzes, Piracicaba, Presidente Prudente, Ribeirão Preto, Santo André, Santos, São Bernardo do Campo, São Carlos, São José do Rio Preto, São José dos Campos, São Paulo e Sorocaba.

Como o primeiro critério de seleção trouxe número baixo de cidades, optou-se por adotar um segundo critério de seleção a fim de obter um modelo matemático mais robusto. $\mathrm{O}$ segundo critério adotado foi a presença de programas de pós-graduação em universidades estaduais e federais no estado: Universidade de São Paulo, USP; Universidade Estadual de Campinas, UNICAMP; Universidade Estadual Paulista, UNESP; Universidade Federal de São Carlos, UFSCAR; Universidade Federal de São Paulo, UNIFESP; Universidade Federal do $\mathrm{ABC}$ (UFABC).

Algumas cidades encontradas estavam presentes na primeira etapa de seleção, estas cidades foram desconsideradas. Por outro lado, 16 novas cidades do estado foram selecionadas: Araçatuba, Araras, Assis, Bauru, Diadema, Franca, Guarulhos, Ilha Solteira, Jaboticabal, Limeira, Lorena, Osasco, Pirassununga, Rio Claro, São Vicente e Tupã.

O Quadro 2 organiza as variáveis por dimensões, códigos utilizados para as análises multivariada de dados, autores e/ou índices os quais utilizaram cada uma delas, e de qual fonte os dados serão extraídos para a aplicação da metodologia e obtenção dos resultados de pesquisa. Todas as bases foram extraídas referente ao ano 2015.

\begin{tabular}{|c|c|c|c|c|}
\hline Dimensão & Código & Variáveis & Referências & Fontes desta pesquisa \\
\hline \multirow{2}{*}{ 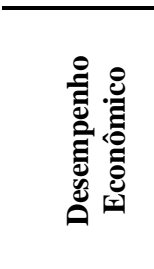 } & PIB & $\begin{array}{l}\text { Produto Interno } \\
\text { Bruno (PIB) per } \\
\quad \text { capita }\end{array}$ & $\begin{array}{l}\text { [2]ALVES et al. (2017); } \\
\text { [10] CASTAÑO et al. (2016); } \\
\text { [34]PIEKKOLA } \quad(2018) ; \\
\text { [44]THANOS et al. (2017); }\end{array}$ & $\begin{array}{l}\text { Sistema Estadual de Análise de Dados } \\
\text { (SEADE) / Informação dos Municípios } \\
\text { Paulistas (IMP) }\end{array}$ \\
\hline & EMP & $\begin{array}{l}\text { Taxa de } \\
\text { empregos } \\
\text { formais }\end{array}$ & $\begin{array}{l}\text { [10] CASTAÑO et al. (2016); } \\
\text { FIRJAN (2014) }\end{array}$ & $\begin{array}{l}\text { Sistema Estadual de Análise de Dados } \\
\text { (SEADE) / Informação dos Municípios } \\
\text { Paulistas (IMP) }\end{array}$ \\
\hline 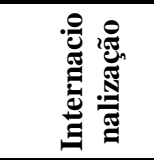 & EXP & $\begin{array}{l}\text { Valor das } \\
\text { Exportações - } \\
\text { FOB (US\$) }\end{array}$ & $\begin{array}{l}\text { [34]PIEKKOLA }(2018) ; \\
\text { [33]OURA et al. (2016); }\end{array}$ & $\begin{array}{l}\text { Sistema Estadual de Análise de Dados } \\
\text { (SEADE) / Informação dos Municípios } \\
\text { Paulistas (IMP) }\end{array}$ \\
\hline 胥 & PAT & $\begin{array}{l}\text { Número de } \\
\text { patentes }\end{array}$ & $\begin{array}{l}\text { [48] WU et al. (2016); } \\
\text { CSC }- \text { Connected SmartCities } \\
\text { (2018); } \\
\text { SÁ PORTO e ROCHA (2017) }\end{array}$ & $\begin{array}{l}\text { Instituto Nacional da Propriedade } \\
\text { Indústria (INPI) }\end{array}$ \\
\hline 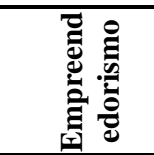 & STA & $\begin{array}{l}\text { Número de } \\
\text { Startups }\end{array}$ & $\begin{array}{l}\text { CSC - Connected SmartCities } \\
\text { (2018) } \\
\text { CIRAVEGNA et al (2018); } \\
\text { MARTIN e JAVALGI (2018) }\end{array}$ & ABStartups/StartupBase \\
\hline
\end{tabular}

\section{Quadro 2 - Dimensões e Variáveis de Pesquisa}

\section{RESULTADOS E DISCUSSÃO}

Para esta pesquisa, assim como para autores mencionados nas etapas de busca de artigos (BRUNECKIENE et al., 2019; LÓPEZ-RUIZ; ALFARO-NAVARRO; NEVADO-PEÑA, 2014; WANG et al, 2018), foi utilizado método quantitativo para elaboração das análises e 
resultados. Como primeiro passo, foram utilizados os dados das 35 cidades selecionadas como amostra e estruturados modelos econométricos utilizando o método de Mínimos Quadrados Ordinários (MQO). O Método dos Mínimos Quadrados Ordinários (MQO) procura encontrar o melhor ajuste para um conjunto de dados tentando minimizar a soma dos quadrados das diferenças entre o valor estimado e os dados observados (HAIR et al.,2010).

Primeiramente foi analisada a relação entre as variáveis dependentes e independentes por meio da matriz de correlação apresentada na Figura 3. Em seguida, foram realizadas regressões lineares simples a fim de verificar a relação de cada variável independente com a variável dependente Produto Interno Bruto (PIB) e Taxas de Emprego (EMP). Pelo fato de a pesquisa considerar as três dimensões: internacionalização, inovação e empreendedorismo, também foi realizada a regressão linear múltipla por mínimos quadrados ordinários (MQO), considerando todas as variáveis independentes em conjunto com a variável dependente PIB, e posteriormente a variável Taxa de Emprego (EMP). A Matriz de Correlação e as Regressões foram feitas com o auxílio do software Stata. Os principais resultados das regressões lineares simples e múltiplas estão representados na Tabela 1.

A fim de reduzir as diferenças de amplitude numérica entre as variáveis selecionadas, foi aplicado logaritmo em toda a base.

Matriz de correlação
\begin{tabular}{l|ccccc}
$($ Obs=35) \\
& EMP & EXP & STA & PIB & PAT \\
\hline EMP & 1.000 & & & & \\
EXP & 0.6683 & 1.000 & & & \\
STA & 0.5600 & 0.7465 & 1.000 & & \\
PIB & 0.5936 & 0.5836 & 0.4922 & 1.000 & \\
PAT & 0.9518 & 0.6579 & 0.5370 & 0.5431 & 1.000
\end{tabular}

\section{Figura 3 - Matriz de Correlação entre as variáveis de pesquisa}

Observa-se que o volume de exportação apresenta correlação com número de patentes $(0,6579)$, taxa de empregos $(0,6683)$, número de startups $(0,7465)$ e PIB $(0,5836)$. Por meio da Matriz de Correlação, nota-se que, entre as dimensões de pesquisa, a "Internacionalização", é a que apresenta maior relação com a variável PIB; a dimensão "Inovação" é a que apresenta maior relação com a variável Taxa de Emprego, seguida pela dimensão "Internacionalização" que também apresenta alta correlação com taxas de empregos. Sendo assim, podem-se observar as três dimensões (internacionalização, empreendedorismo e inovação) apresentando correlação com a dimensão "Desenvolvimento Econômico", conforme sugerido na hipótese de pesquisa.

As regressões lineares simples entre as variáveis explicativas com Produto Interno Bruto (PIB) apresentaram baixo coeficiente de determinação $\left(\mathrm{R}^{2}\right)$ e coeficiente de determinação ajustado $\left(\mathrm{R}^{2}\right)$ ajustado. Porém, ao analisar a regressão linear simples entre as variáveis EXP e PIB verifica-se maior coeficiente de determinação $\left(R^{2}\right)-34,06 \%$ e coeficiente de determinação ajustado $-32,07$. O teste $t$ demonstra relação significativa entre volume de exportação e produto interno bruto (PIB) - 3,25 (acima de 2, conforme sugere Hair et al. (2010)).

Por outro lado, as correlações lineares simples entre as variáveis explicativas e Taxas de Empregos Formais, apresentaram resultados mais significativos. Ao analisar a relação entre STA e EMP verifica-se coeficiente de determinação $\left(R^{2}\right)$ e $R^{2}$ ajustado, respectivamente, 
$31,36 \%$ e $29,28 \%$. A constatação de relação entre as variáveis é confirmada ao ser verificado o teste t, o qual se apresenta acima de 2, ou seja, conforme Hair et al. (2010) significativo. As variáveis EXP e EMP apresentam coeficiente de determinação $\left(\mathrm{R}^{2}\right) 44,66 \%$ e coeficiente de determinação ajustado $42,98 \%$. O teste t demonstra relação significativa entre volume de exportação e taxa de empregos - 5,16 (acima de 2, conforme Hair et al. (2010)).

\begin{tabular}{|c|c|c|c|c|}
\hline Variáveis (Dimensões) & $\begin{array}{l}\text { Matriz de } \\
\text { Correlação }\end{array}$ & $\mathbf{R}^{2}$ Ajustado & Test t & P-Valor \\
\hline $\begin{array}{l}\text { Número de Startups (Empreendedorismo) x } \\
\text { Taxa de Emprego (Desempenho Econômico) }\end{array}$ & 0,5600 & 0,2928 & 3,88 & 0,0000 \\
\hline $\begin{array}{l}\text { Número de Startups (Empreendedorismo) x } \\
\text { Quantidade de Patentes (Inovação) }\end{array}$ & 0,5370 & NA & NA & NA \\
\hline $\begin{array}{l}\text { Volume de Exportação (Internacionalização) } \\
\text { x Taxa de Emprego (Desempenho } \\
\text { Econômico) }\end{array}$ & 0,6683 & 0,4298 & 5,16 & 0,0000 \\
\hline $\begin{array}{l}\text { Número de Startups (Empreendedorismo) x } \\
\text { Volume de Exportação (Internacionalização) }\end{array}$ & 0,7465 & NA & NA & NA \\
\hline $\begin{array}{l}\text { Quantidade de Patentes (Inovação) x } \\
\text { Volume de Exportação (Internacionalização) }\end{array}$ & 0,6579 & NA & NA & NA \\
\hline $\begin{array}{l}\text { Volume de Exportação (Internacionalização) } \\
\text { x PIB (Desempenho Econômico) }\end{array}$ & 0,5836 & 0,3207 & 4,13 & 0,002 \\
\hline $\begin{array}{l}\text { Quantidade de Patentes (Inovação), Volume } \\
\text { de Exportação (Internacionalização), } \\
\text { Número de Startups (Inovação) x PIB } \\
\text { (Desempenho Econômico) }\end{array}$ & NA & 0,3306 & $\begin{array}{l}1,39(\text { EXP) } \\
1,46 \text { (PAT) } \\
0,47 \text { (STA) }\end{array}$ & $\begin{array}{l}0,174(\text { EXP) } \\
0,155 \text { (PAT) } \\
0,642 \text { (STA) }\end{array}$ \\
\hline $\begin{array}{l}\text { Quantidade de Patentes (Inovação), Volume } \\
\text { de Exportação (Internacionalização), } \\
\text { Número de Startups (Inovação) x Taxa de } \\
\text { Emprego (Desempenho Econômico) }\end{array}$ & NA & 0,9013 & $\begin{array}{c}0,47(\mathrm{EXP}) \\
12,52(\mathrm{PAT}) \\
0,55(\mathrm{STA})\end{array}$ & $\begin{array}{l}0,640 \text { (EXP) } \\
0,000 \text { (PAT) } \\
0,585 \text { (STA) }\end{array}$ \\
\hline
\end{tabular}

Tabela 1 - Síntese de Resultados (Matriz de Correlação, $\mathbf{R}^{2}$ Ajustado, Teste t, e P-Valor)

Para iniciar as análises da regressão linear múltipla entre a variável dependente PIB e as variáveis independentes STA, EXP, PAT e a variável dependente EXP e as variáveis independentes STA, EXP, PAT, primeiramente foi observada a qualidade de ajustamento do modelo. Como a soma dos quadrados residuais se apresentou menor do que a soma dos quadrados explicados e a soma dos quadrados totais, considerou-se os modelos estão ajustados. Foram realizadas 35 observações (dados das 35 cidades da amostra), e o teste de distribuição f apresentou-se maior do que 5, indicando que os modelos estão ajustados (HAIR et al., 2010).

Conforme apresentado na Tabela 1 , o coeficiente de determinação $\left(\mathrm{R}^{2}\right), 38,96 \%$ da variação do PIB pode ser explicada pelo número de startups (STA), valor das exportações (EXP) e número de patentes (PAT). Conforme Hair et al. (2010), ao ser mais criterioso quanto ao coeficiente de determinação de uma regressão linear múltipla - ou seja, regressão linear que possua mais de uma variável explicativa - deve-se considerar o coeficiente de determinação ajustado ( $\mathrm{R}^{2}$ ajustado). Seguindo essa regra, pode-se afirmar que 33,06\% da variação do PIB podem ser explicados pelas variáveis independentes. Ao analisar o teste t, verifica-se que as variáveis não possuem coeficiente significativo - conforme a regra, menor do que 2 (HAIR, et al. 2010). Além disso, todos os P-valores apresentaram-se maiores do que 0,05, ou seja, 
conforme a literatura os resultados não foram significativos. O coeficiente de determinação $\left(\mathrm{R}^{2}\right)$ 91,00\% da variação da Taxa de Empregos Formais pode ser explicada pelo número startups (STA), valor das exportações (EXP) e número de patentes (PAT). Conforme Hair et al. (2010), seguindo a regra de utilização do $\mathrm{R}^{2}$ ajustado, pode-se afirmar que $90,13 \%$ da variação da taxa de emprego pode ser explicada pelas variáveis independentes.

Para verificar se os modelos elaborados nesta pesquisa apresentam ou não problema de multicolinearidade, foi feita a análise do Fator de Inflação de Variância (FIV). O FIV mede o quanto da variância de cada coeficiente de regressão do modelo estatístico se encontra inflado em relação à situação em que as variáveis independentes não estão correlacionadas. Conforme a literatura (HAIR et al., 2010), se as variáveis explicativas apresentarem FIV maior do que 10, há presença de multicolinearidade. Portanto, conforme evidenciado nas Figuras 4, os modelo criados nesta pesquisa não apresentam problema de multicolinearidade, pois a variável com maior FIV é 2,86 .

Fator de Inflação da Variância (FIV)

\begin{tabular}{c|cc} 
Variável & FIV & $1 /$ FIV \\
\hline EXP & 2.86 & 0.349919 \\
STA & 2.28 & 0.439030 \\
PAT & 1.78 & 0.562427 \\
\hline Média FIV & 2.30 &
\end{tabular}

Figura 4 - Fator de Inflação da Variância (FIV)

Outro teste realizado para o modelo foi o teste de heterocedasticidade. Este teste tem como hipótese que a variância dos resíduos gerados pela estimação do modelo seja constante. Se isso ocorrer, o pressuposto de homocedasticidade é satisfeito (HAIR et al., 2010). Conforme evidenciado na Figura 5, ao ser realizado o teste para o modelo considerando a variável PIB como variável dependente, a hipótese não foi rejeitada, uma vez que chi está diferente de zero. Sendo assim, para este modelo não há problema de heterocedasticidade. Na Figura 6, ao ser realizado o teste para o modelo considerando a variável Taxa de Empregos Formais (EMP) como variável dependente, a hipótese também não foi rejeitada, uma vez que chi está diferente de zero. Sendo assim, para este modelo não há problema de heterocedasticidade.

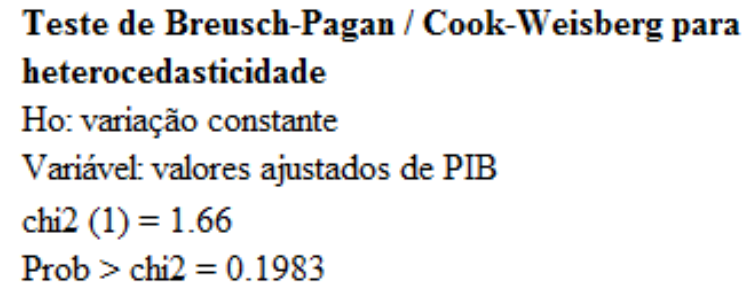

Figura 5 - Heterocedasticidade - PIB como variável dependente

\author{
Teste de Breusch-Pagan / Cook-Weisberg para \\ heterocedasticidade \\ Ho: variação constante \\ Variável: valores ajustados de EMP \\ $\operatorname{chi} 2(1)=2.72$ \\ Prob $>$ chi $2=0.0988$
}
Figura 6 - Heterocedasticidade - EMP como variável dependente

\section{CONCLUSÃO}

Ao observar a matriz de correlação (Figura 3), conforme constatado na revisão da literatura, nota-se que há relação entre as variáveis dependentes de pesquisa; A dimensão 
empreendedorismo correlacionada com a dimensão internacionalização, conforme constatado na literatura (CIRAVEGNA; MAJANO; ZHAN, 2014; DAI et al, 2016; MARTIN; JAVALGI, 2018; OPARAOCHA, 2015; VEGLIO; ZUCCHELLA, 2015; ZHANG et al., 2016); A dimensão empreendedorismo correlacionada com a dimensão inovação (LEWANDOWSKA; SZYMURA-TYC; GOŁEBIOWSKI, 2016; NATÁRIO; BRAGA; FERNANDES, 2018; OPAROACHA, 2015); A dimensão inovação correlacionada com a dimensão internacionalização (BOERMANS; ROELFSEMA, 2015, 2016; CASTAÑO; MÉNDEZ; GALINDO, 2016; CHIVA; GHAURI; ALEGRE, 2014; LEWANDOWSKA; SZYMURATYC; GOŁEBIOWSKI, 2016; OURA; ZILBER; LOPES, 2016; VEGLIO; ZUCCHELLA, 2015; WU et al., 2016). Ao observar a Tabela 1, nota-se que entre os resultados mais expressivos está a regressão linear múltipla com a variável dependente Taxa de Empregos Formais. O modelo elaborado apresentou F maior do que 5, ou seja, modelo ajustado, e os testes de multicolineariade de heterocedasticidade não apresentaram problemas. Ainda assim, o R ${ }^{2}$ ajustado foi alto - 90,13, evidenciando alta influência das variáveis explicativas na variável dependente Taxa de Empregos Formais.

As cidades que apresentam maior taxa de emprego do estado - São Paulo e Campinas possuem diferenciais que as auxiliam empreendedores. Ao entrar no site da prefeitura de São Paulo, é possível ter acesso à agência de promoção de investimentos e exportação do município, a qual prospecta investigadores para negócios, capacita pequenas e médias empresas por meio de diversos programas e apoia o desenvolvimento de startups. Além disso, a prefeitura disponibiliza o passo a passo de como abrir empresas, oferece informações de programas e benefícios, alvarás, certidões, licenças, legislação, e ainda faz um compilado das principais oportunidades de negócios. Ao acessar o site da prefeitura de Campinas é possível ter acesso a alvarás, certidões, licenças, atendimento sobre tributos, a publicações e informações jurídicas, consulta CNAE, e outros. Além disso, as duas cidades contam com a presença de universidades como a Universidade de São Paulo (USP) e a Universidade Estadual de Campinas (UNICAMP), que fornecem cursos de graduação e pós-graduação e, consequentemente, capital qualificado para exercer atividades internacionais, empreendedoras e inovadoras. Outras instituições como a INOVA, agência de inovação da Unicamp, estimulam a inovação, o empreendedorismo e, consequentemente, o desenvolvimento econômico.

Outras cidades com PIB per capita elevado, como em Piracicaba, contam com a presença da Universidade de São Paulo (USP-Esalq), o curso de odontologia da Universidade Estadual de Campinas (UNICAMP), entre outras universidades como a Universidade Metodista de Piracicaba (UNIMEP), e parques tecnológicos ligados à atividade canavieira da região. Porém a cidade não consta no Mapa SP Conecta com incubadoras e centros de P\&D.

Conforme resultados apresentados nesta pesquisa: legitimação da importância de atividades internacionais, empreendedoras e inovadoras para a taxa de empregos formais, e, em vista da importância da teoria institucional notada na revisão da literatura, formuladores de políticas públicas devem desafiar-se em como apoiar atividades internacionais, empreendedoras e inovadoras por meio de apoio institucional e, assim, obter melhores taxas de emprego do país. Portanto, respondendo à questão de pesquisa e atendendo ao objetivo proposto, conclui-se que a internacionalização, empreendedorismo e inovação estão correlacionados entre si no contexto das cidades e apresentam correlação significativa com a dimensão desempenho econômico, principalmente com a variável taxa de emprego. Desta 
forma, formuladores de políticas públicas devem preocupar-se em empregar políticas públicas que diminuam a percepção de incerteza dos empreendedores para investir no Brasil. Empreendedores reconhecem que mercados emergentes, como o Brasil, são bons lugares para investimentos e, portanto utilizam de estratégias políticas para reduzir incertezas. (HEIDENREICH; MOHR; PUCK, 2015). Estudos elaborados na China apontam que os recursos referentes aos laços políticos se mostraram mais importantes do que laços entre firmas para o estímulo à inovação e internacionalização (ZHANG et al., 2016). Além disso, apresentam boas práticas: o governo da China frequentemente apoia firmas inovadoras a expandir seus negócios e reduz custos de pesquisa e desenvolvimento (P\&D) por meio de regulamentações, redução de impostos e concessão de créditos em bancos (ZHANG et al., 2016).

\section{$6 \quad$ REFERÊNCIAS}

ABDALA, L. N. et al. Centro de Inovação: alinhamento conceitual. Florianópolis: Perse, 2016. [1]AHLSTROM, D. et al. A global perspective of entrepreneurship and innovation in China. Multinational Business Review, v. 26, n. 4, p. 302-318, 2018.

[2]ALVES, M. F. R. et al. Internationalization and innovation in nascent companies: does gender matter? Journal of Small Business and Enterprise Development, v. 24, n. 4, p. 887-905, 2017.

ASSOCIAÇÃO BRASILEIRA DE STARTUPS (ABStartups). STARTUPBASE, 2019. Disponível em: https://startupbase.com.br. Acesso em: 15 jun. 2019.

BARON, R.A.; SHANE, S.A. Empreendedorismo: uma visão de processo. São Paulo: Cengage Learning, 2010.

[3]BARONCHELLI, G.; CASSIA, F. Exploring the antecedents of born-global companies' international development. International Entrepreneurship and Management Journal, v. 10, n. 1, p. 67-79, 2014.

[4]BASTIAN, B. L.; TUCCI, C. L. Entrepreneurial advice sources and their antecedents: Venture stage, innovativeness and internationalization. Journal of Enterprising Communities, v. 11, n. 2, p. 214-236, 2017.

[5]BAUM, M.; SCHWENS, C.; KABST, R. A latent class analysis of small firms' internationalization patterns. Journal of World Business, v. 50, n. 4, p. 754-768, 2015.

[6]BIANCHI, C.; GLAVAS, C.; MATHEWS, S. SME international performance in Latin America: The role of entrepreneurial and technological capabilities. Journal of Small Business and Enterprise Development, v. 24, n. 1, p. 176-195, 2017.

[7]BOERMANS, M. A.; ROELFSEMA, H. Small firm internationalization, innovation, and growth. International Economics and Economic Policy, v. 13, n. 2, p. 283-296, 2016.

[8]BOERMANS, M. A.; ROELFSEMA, H. The Effects of Internationalization on Innovation: Firm-Level Evidence for Transition Economies. Open Economies Review, v. 26, n. 2, p. 333$350,2015$.

BRUNECKIENE, J. et al. An Assessment of Socio-Economic Systems' Resilience to Economic Shocks: The Case of Lithuanian Regions. Sustainability, v. 11, n. 3, p. 566, 22 jan. 2019. 
[9]BRUNEEL, J.; CLARYSSE, B.; AUTIO, E. The role of prior domestic experience and prior shared experience in young firm internationalization. International Small Business Journal: Researching Entrepreneurship, v. 36, n. 3, p. 265-284, 23 maio 2018.

[10]CASTAÑO, M.-S.; MÉNDEZ, M.-T.; GALINDO, M.-Á. Innovation, internationalization and business-growth expectations among entrepreneurs in the services sector. Journal of Business Research, v. 69, n. 5, p. 1690-1695, 2016.

[11]CHE SENIK, Z. et al. A Model for Understanding SMEs Internationalization in Emerging Economies. Jurnal Pengurusan, v. 41, p. 25-42, 1 set. 2014.

[12]CHIVA, R.; GHAURI, P.; ALEGRE, J. Organizational Learning, Innovation and Internationalization: A Complex System Model. British Journal of Management, v. 25, n. 4, p. 687-705, 2014.

[13]CIRAVEGNA, L. et al. The antecedents of early internationalization: A configurational perspective. International Business Review, v. 27, n. 6, p. 1200-1212, 2018.

[14]CIRAVEGNA, L.; MAJANO, S. B.; ZHAN, G. The inception of internationalization of small and medium enterprises: The role of activeness and networks. Journal of Business Research, v. 67, n. 6, p. 1081-1089, 2014.

[15]CUERVO-CAZURRA, A.; RAMAMURTI, R. Home country underdevelopment and internationalization: Innovation-based and escape-based internationalization. Competitiveness Review, v. 27, n. 3, p. 217-230, 2017.

[16]DAI, L. et al. Entrepreneurial orientation and international scope: The differential roles of innovativeness, proactiveness, and risk-taking. Journal of Business Venturing, v. 29, n. 4, p. 511-524, 2014.

[17]DÍAZ-ARIZA, D. M.; GARCÍA-CASTIBLANCO, C. P.; MUÑOZ, C. A. P. Government programs for business internationalization: A reflection upon the case of the cosmetics and toiletries sector in Bogota | Les programmes gouvernementaux pour l'internationalisation des entreprises: Une réflexion du cas du secteur des cosmétique. Innovar, v. 29, n. 71, p. 69-78, 2019.

ENSSLIN, L.; ENSSLIN, S. R.; LACERDA, R. T. O.; TASCA, J. E. ProKnow-C, knowledge Development Process-Constructivist. Processo técnico com patente de registro pendente junto ao INPI. Brasil, 2010.

FEDERAÇÃO DAS INDÚSTRIAS DO RIO DE JANEIRO (FIRJAN). Índice FIRJAN de Desenvolvimento Municipal (IFDM), 2014.

[18]FELZENSZTEIN, C. et al. Networks, Entrepreneurial Orientation, and Internationalization Scope: Evidence from Chilean Small and Medium Enterprises. Journal of Small Business Management, v. 53, p. 145-160, 2015.

FUNDAÇÃO JOSÉ PINHEIRO. Índice Mineiro de Responsabilidade Social (IMRS). Disponível em: http://imrs.fjp.mg.gov.br/. Acesso em: 26 jan. 2019.

GUIMARÃES, S. K.; AZAMBUJA, L. R. Internacionalização de micro, pequenas e médias empresas inovadoras no Brasil - Desafios e novo paradigma de desenvolvimento. 2018.

HAIR, J. ANDERSON, R. BLACK, W. BABIN, J. B. Análise Multivariada de Dados. $7^{\text {a }}$ Edição, 2010

[19]HAGEN, B.; ZUCCHELLA, A. Born Global or Born to Run? The Long-Term Growth of Born Global Firms. Management International Review, v. 54, n. 4, p. 497-525, 2014. 
[20]HALILEM, N.; AMARA, N.; LANDRY, R. Exploring the relationships between innovation and internationalization of small and medium-sized enterprises: A nonrecursive structural equation model. Canadian Journal of Administrative Sciences, v. 31, n. 1, p. 18-34, 2014.

[21]HEIDENREICH, S.; MOHR, A.; PUCK, J. Political strategies, entrepreneurial overconfidence and foreign direct investment in developing countries. Journal of World Business, v. 50, n. 4, p. 793-803, 2015.

[22]HERSTAD, S. J.; EBERSBERGER, B. On the Link between Urban Location and the Involvement of Knowledge-Intensive Business Services Firms in Collaboration Networks. Regional Studies, v. 49, n. 7, p. 1160-1175, 2015.

INSTITUTO DE PESQUISA ECONÔMICA APLICADA (IPEA). Disponível em: http://www.ipea.gov.br/acessoainformacao. Acesso em 09 out. 2019.

INVESTE SÃO PAULO. Disponível em: https://www.investe.sp.gov.br/por-que-sp/economiadiversificada/pib/. Acesso em: 02 fev. 2019.

JOHANSON, J.; VAHLNE, J. E. The internationalization process of the firm - a model of knowledge development and increasing foreign market commitments. Journal of International Business Studies, 1977.

[23]KALINIC, I.; SARASVATHY, S. D.; FORZA, C. "Expect the unexpected": Implications of effectual logic on the internationalization process. International Business Review, v. 23, n. 3, p. 635-647, 2014.

[24]KAUR, S.; SANDHU, M. S. Internationalisation of born global firms: Evidence from Malaysia. Journal of the Asia Pacific Economy, v. 19, n. 1, p. 101-136, 2014.

KHAN, S.; ZAMAN, A. U. Future cities: Conceptualizing the future based on a critical examination of existing notions of cities. Cities, v. 72, p. 217-225, fev. 2018.

[25]KURYAN, N.; KHAN, M. S.; GUSTAFSSON, V. Born globals and business incubators: a case analysis. International Journal of Organizational Analysis, v. 26, n. 3, p. 490-517, 2018. [26]LEWANDOWSKA, M. S.; SZYMURA-TYC, M.; GOŁEBIOWSKI, T. Innovation complementarity, cooperation partners, and new product export: Evidence from Poland. Journal of Business Research, v. 69, n. 9, p. 3673-3681, 2016.

LÓPEZ-RUIZ, V.-R.; ALFARO-NAVARRO, J.-L.; NEVADO-PEÑA, D. Knowledge-city index construction: An intellectual capital perspective. Expert Systems with Applications, v. 41, n. 12, p. 5560-5572, set. 2014.

[27]MARTIN, S. L.; JAVALGI, R. R. G. Epistemological foundations of international entrepreneurship. International Entrepreneurship and Management Journal, v. 14, n. 3, p. 671680, 2018.

[28]MILANOV, H.; FERNHABER, S. A. When do domestic alliances help ventures abroad? Direct and moderating effects from a learning perspective. Journal of Business Venturing, v. 29, n. 3, p. 377-391, maio 2014.

[29]MUSTEEN, M.; DATTA, D. K.; BUTTS, M. M. Do International Networks and Foreign Market Knowledge Facilitate SME Internationalization? Evidence From the Czech Republic. Entrepreneurship: Theory and Practice, v. 38, n. 4, p. 749-774, 2014.

NATÁRIO, M. M. S.; BRAGA, A. M.; FERNANDES, G. P. Determinants of entrepreneurial and innovation performance in cross-border regions $\mid$ Les déterminants de la performance 
entrepreneuriale et innovante dans les régions transfrontalières | Determinantes do desempenho empreendedor e inovador nas regiões tran. Innovar, v. 28, n. 70, p. 39-54, 2018.

NAVARRO-GARCÍA, A.; REY-MORENO, M.; BARRERA-BARRERA, R. Compromiso, Recursos, Emprendimiento Exportador Y Resultados Empresariales. Revista de Administração de Empresas, v. 57, n. 2, p. 135-147, 2017.

[30]NORDMAN, E. R.; TOLSTOY, D. The impact of opportunity connectedness on innovation in SMEs' foreign-market relationships. Technovation, v. 57-58, p. 47-57, nov. 2016.

NUNES, M. P.; STEINBRUCH F. K. Internacionalização e a necessidade de inovação em modelo de negócios - uma abordagem teórica. 2018.

[31]NYUUR, R. B.; BRECIC, R.; DEBRAH, Y. A. SME international innovation and strategic adaptiveness: The role of domestic network density, centrality and informality. International Marketing Review, v. 35, n. 2, p. 280-300, 2018.

[32]OPARAOCHA, G. O. SMEs and international entrepreneurship: An institutional network perspective. International Business Review, v. 24, n. 5, p. 861-873, 2015.

[33]OURA, M. M.; ZILBER, S. N.; LOPES, E. L. Innovation capacity, international experience and export performance of SMEs in Brazil. International Business Review, v. 25, n. 4, p. $921-$ 932, 2016.

OVIATT, B. M.; McDougall, P. P. Toward a theory of international new ventures. Journal of International Business Studies, 1994.

[34]PIEKKOLA, H. Internationalization via export growth and specialization in Finnish regions. Cogent Economics and Finance, v. 6, n. 1, p. 1-25, 2018.

[35]PIERRE, J. Multilevel governance as a strategy to build capacity in cities: Evidence from Sweden. Journal of Urban Affairs, v. 41, n. 1, p. 103-116, 2019.

[36]PRANGE, C.; PINHO, J. C. How personal and organizational drivers impact on SME international performance: The mediating role of organizational innovation. International Business Review, v. 26, n. 6, p. 1114-1123, dez. 2017.

[37]RASK, M. Internationalization through business model innovation: In search of relevant design dimensions and elements. Journal of International Entrepreneurship, v. 12, n. 2, p. 146$161,2014$.

[38]REN, S.; EISINGERICH, A. B.; TSAI, H.-T. How do marketing, research and development capabilities, and degree of internationalization synergistically affect the innovation performance of small and medium-sized enterprises (SMEs)? A panel data study of Chinese SMEs. International Business Review, v. 24, n. 4, p. 642-651, 2015.

[39]RIALP-CRIADO, A.; KOMOCHKOVA, K. Innovation strategy and export intensity of Chinese SMEs: The moderating role of the home-country business environment. Asian Business and Management, v. 16, n. 3, p. 158-186, 2017.

[40]RODIL, Ó.; VENCE, X.; SÁNCHEZ, M. D. C. The relationship between innovation and export behaviour: The case of Galician firms. Technological Forecasting and Social Change, v. 113 , p. 248-265, 2016.

[41]ROSENBAUM, G. O. Female entrepreneurial networks and foreign market entry. Journal of Small Business and Enterprise Development, v. 24, n. 1, p. 119-135, 2017.

SÁ PORTO, P. C.; ROCHA, F. M. M. City size and competitiveness for the cities of São Paulo: a spatial statistics analysis. Revista Brasileira de Inovação, v. 17, n. 1, p. 63, 2017. 
SANTOS, D.; JESUS, V. DE. A arte do encontro: a paradiplomacia e a internacionalização das cidades criativas. p. 51-76, 2017.

SERVIÇO BRASILEIRO DE APOIO ÀS MICRO E PEQUENAS EMPRESAS. Índice de Desenvolvimento de Micro e Pequena Empresa (IDMPE). Disponível em: http://app.pr.sebrae.com.br/idmpe. Acesso em: 26 jan. 2019.

SCHUMPETER, J.A. O capitalismo pode sobreviver? São Paulo: Abril Cultural, 1952.

SCHUMPETER, J.A. A teoria do desenvolvimento econômico. São Paulo: Nova Cultural, 1988.

[42]SIEDSCHLAG, I.; ZHANG, X. Internationalisation of firms and their innovation and productivity. Economics of Innovation and New Technology, v. 24, n. 3, p. 183-203, 2015.

[43]SWOBODA, B.; OLEJNIK, E. Linking Processes and Dynamic Capabilities of International SMEs: The Mediating Effect of International Entrepreneurial Orientation. Journal of Small Business Management, v. 54, n. 1, p. 139-161, 2016.

[44]THANOS, I. C.; DIMITRATOS, P.; SAPOUNA, P. The implications of international entrepreneurial orientation, politicization, and hostility upon SME international performance. International Small Business Journal: Researching Entrepreneurship, v. 35, n. 4, p. 495-514, 2017.

[45]VANNINEN, H.; KUIVALAINEN, O.; CIRAVEGNA, L. Rapid multinationalization: Propositions for studying born micromultinationals. International Business Review, v. 26, n. 2, p. 365-379, abr. 2017.

[46]VEGLIO, V.; ZUCCHELLA, A. Entrepreneurial firms in traditional industries. Does innovation matter for international growth? Journal of International Entrepreneurship, v. 13, n. 2, p. 138-152, 2015.

WANG, N. et al. Evaluation of Urban circular economy development: An empirical research of 40 cities in China. Journal of Cleaner Production, v. 180, p. 876-887, abr. 2018.

[47]WILCHES TORRES, L. D.; NIÑO AMÉZQUITA, J. L. Territorial internationalization and competitiveness in Latin America. A medium-sized city perspective | Internacionalización territorial y competitividad en América Latina. Una perspectiva desde las ciudades intermedias. Espacios, v. 38, n. 54, 2017.

[48]WU, J. et al. Internationalization and innovation performance of emerging market enterprises: The role of host-country institutional development. Journal of World Business, v. 51, n. 2, p. 251-263, 2016.

[49]ZHANG, X. et al. What drives the internationalization of Chinese SMEs? The joint effects of international entrepreneurship characteristics, network ties, and firm ownership. International Business Review, v. 25, n. 2, p. 522-534, 2016. 